\title{
Google Search+ Your World ¿Búsqueda Social o Asocial?
}

\author{
Esmeralda Serrano Mascaraque \\ Rosa Pintado-Grande
}

Recibido: 14.06.2012

Aceptado: 05.07.2012

\section{Resumen}

El dinamismo de la web lleva consigo una evolución en la búsqueda y recuperación de información, intentando acercarse al carácter social de éstas. La proliferación de las redes sociales es la principal causante de la versatilidad, dispersión y naturaleza efímera de la información en la web. La inteligencia colectiva que tilda esta nueva época, genera la aparición de nuevas herramientas de recuperación como el nuevo buscador de Google que propone una recuperación más social y los incipientes cambios en el algoritmo de su motor de búsqueda para hacerlo más semántico. Y, por último, demanda ciertos perfiles profesionales capaces de gestionar esta nueva información.

\section{Palabras clave}

Google Search Plus Your World, Web 2.0, Web semántica, Búsqueda social, Recuperación de Información, Redes Sociales.

\section{Abstract}

The dynamism of the web brings with it an evolution in the search and retrieval of information, trying to approach the social character of these. The proliferation of social networking is the main cause of the versatility, dispersion and transient nature of the information on the web. Collective intelligence which labels this new era, creates the appearance of new recovery tools like Google's new search engine that offers a social recovery and the incipient changes in the algorithm for its search engine to make it more semantic. And finally, demand certain professional profiles able to manage this new information

\section{Keywords}

Google Search Plus Your World, Web 2.0, Semantic Web, Social Search, Information Retrieval, Social Networks. 


\section{INTRODUCCIÓN}

El dinamismo de la World Wide Web demanda unas nuevas herramientas capaces de gestionar eficazmente estos incipientes y versátiles recursos informativos de la nueva red social, la cual pone especial énfasis en la colaboración on line, la interactividad y la posibilidad de compartir recursos entre sus usuarios. Por ello, Google ha lanzado su nuevo y polémico buscador personalizado Google Search Plus Your World. Éste nos permite acceder no sólo a los contenidos creados de forma pública en Internet, sino a aquéllos publicados por nuestros contactos y relacionados con nuestro perfil social en Google + .

La Web nació de la mano de Tim Berners-Lee, el inventor de hipertexto. Trivializando, al principio, ésta sólo consistía en imágenes y textos colgados en un ordenador conectado a la red (servidor); para permitir el acceso a las imágenes o a los textos se debía acceder a ese ordenador y reemplazarlo manualmente. Cuando la web empezó a popularizarse el concepto era simple pero poderoso: podía publicarse información a la vista de cualquiera que tuviera un ordenador y una línea de teléfono. Las empresas comenzaron a interesarse en acceder a este sistema y comenzaron a vislumbrarse muchas posibilidades.

Fue el momento de las .com, a las empresas les bastaba con agregar una e- al principio o un .com al final de sus nombres para cotizar mejor en bolsa, fue el gran furor de la tecnología de la comunicación, la sensación de algo nuevo y poderoso que se transformó en una burbuja que reventó a finales del 2001 generando una crisis económica en las empresas de Tecnología de la información y luego propagándose a otros ambientes. A esa etapa se la denominó Web 1.0.

Paralelamente a esto, comenzaron a surgir aplicaciones server-side; es decir, programas que funcionaban en los servidores que alojan las páginas Web's e interactúan con ellas.

Además, comenzaron a aparecer los estándares XHTML, CSS, Javascript, que posibilitaron una Web más rica, funcional y usable. Estas tecnologías en conjunto permitieron que los usuarios participaran con la Web, enviando datos ellos mismos, y eran las tecnologías server-side las encargadas de modificar el contenido de las páginas.

El término 2.0 está asociado con un fenómeno social basado en la interacción que se logra a partir de diferentes aplicaciones en la Web, que facilitan el compartir información, la interoperabilidad y el diseño centrado en el usuario. La Web 2.0 es una actitud colaborativa. 


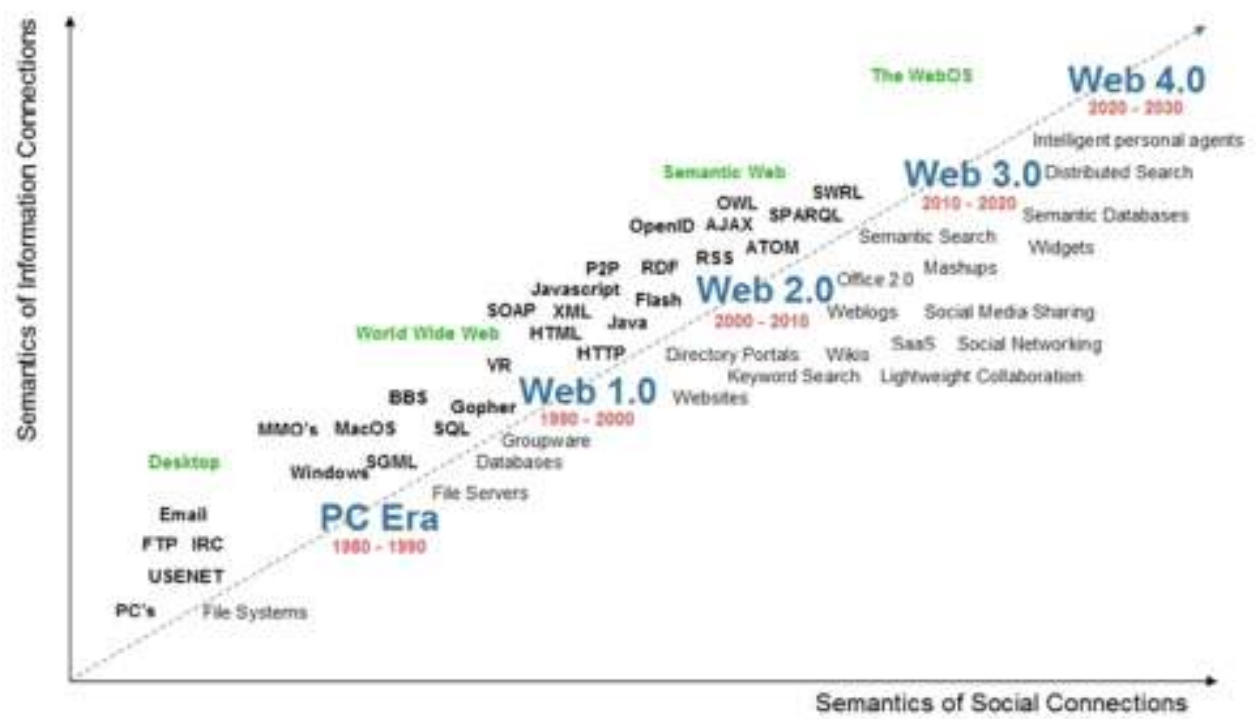

Fuente: Radar Networks \& Nova Spivack, 2007 - www.radarnetworks.com

A continuación, se enumeran los principales servicios ofrecidos por la Web 2.0:

Blogs. El término web-log lo acuñó Jorn Barger en 1997 para referirse a un diario personal en línea de actualización constante por parte de su autor. Más adelante, las dos palabras Web y Log, se comprimieron para formar una sola, Weblog y ésta derivó en una ostensiblemente más corta, Blog.

Un blog es un sitio Web que facilita la publicación instantánea de entradas o posts, posibilitando una retroalimentación en forma de comentarios.

El acto de escribir un blog se conoce como blogging; y por extensión, una persona que escribe un blog es un blogger.

Wikis. Una Wiki (etimológicamente proviene del hawaiano y significa rápido o veloz) es una página Web o un conjunto de ellas que cualquier persona, a quién se le permita el acceso, puede editar fácilmente desde cualquier lugar. En pocas palabras, es un sitio Web de construcción colectiva, con un tema específico, en el cual los usuarios tienen libertad para añadir, eliminar o editar los contenidos.

Social Bookmarking. Los sistemas de social bookmarking o etiquetado social comparten una serie de características comunes. Permiten a los usuarios crear listas de marcadores o favoritos que pueden almacenarse centralmente en un servicio remoto, en lugar de hacerlo en el navegador del usuario. Además, permite que éstos se compartan con otros usuarios del sistema y precisamente en eso consiste su connotación social. A estos marcadores se les pueden añadir también etiquetas mediante palabras clave; y es importante resaltar una diferencia sustancial con el uso de la categorización tradicional en base a carpetas de las listas de favoritos del navegador: un marcador puede pertenecer a más de una categoría.

Intercambio Multimedia. Una de las mayores áreas de crecimiento en la Web se ha dado en servicios que facilitan almacenar y compartir contenido multimedia. Ejemplos bastante 
conocidos son YouTube (vídeos), Flickr (fotografías) y Odeo (podcasts). Estos servicios, que tienen amplia acogida, se han apropiado de la idea de la Web para escritores (writable web) donde los usuarios no solamente consumen contenido, sino que contribuyen activamente en la producción de éste en la Web y lo hacen a escala masiva. En la actualidad, son literalmente millones de personas las que participan en compartir e intercambiar productos multimedia; produciendo sus propios podcasts, vídeos, fotografías, presentaciones, documentos, etc.

Podcasting. La palabra podcast proviene de la contracción de los términos iPod (reproductor MP3 de Apple) y broadcast (emisión). Inicialmente hacían referencia a grabaciones de audio utilizadas para almacenar audio streams en los incipientes blogs que en su momento se llamaron audio blogs. Más recientemente, el término se amplió para incluir las grabaciones de vídeo, que se llamaron vídeopodcast. Un podcast se hace generando, mediante una grabadora digital de voz o un aparato similar, un archivo de audio en formato MP3 que se pueda reproducir tanto en un PC como en una amplia gama de aparatos portátiles que acepten este formato (iPod, teléfonos móviles, equipos de sonido, memorias USB, etc.) Posteriormente el archivo se aloja en un servidor y se comunica al mundo su existencia utilizando Really Simple Sindication. Los usuarios de podcasts se suscriben a un servicio de RSS feeds o agregadores y reciben información permanente sobre nuevos podcasts a medida que éstos vayan estando disponibles.

Sindicación. EI RSS (Really Simple Sindication) hace referencia a un estándar de sindicación. Esto es a una familia de formatos de datos conocidos como feed, Web feed o channel utilizados para ofrecer a los usuarios información de contenido que se actualiza con mucha frecuencia, tales como entradas de Blogs, titulares de medios o podcasts. El RSS se compone de dos elementos:

El código XML que ofrecen los sitios Web como un beneficio adicional para sus lectores/usuarios (en la actualidad casi todas las páginas Web lo han implementado) y una aplicación especial conocida como lector de RSS o agregador que debe tener quien desee hacer uso de él. Así, una persona puede utilizar un programa lector de RSS para suscribirse a los sitios Web de su interés que ofrezcan este servicio y estar permanentemente informada, automáticamente, de los contenidos nuevos que se publiquen en cualquiera de esos sitios.

Redes Sociales. En 2002 comienzan a aparecer sitios Web promocionando las redes de círculos de amigos en línea, cuando el término se empleaba para describir las relaciones en las comunidades virtuales, y se hizo popular en 2003 con la llegada de sitios tales como MySpace o Xing. Hay más de 200 sitios de redes sociales, aunque Friendster ha sido uno de los que mejor ha sabido emplear la técnica del círculo de amigos. La popularidad de estos sitios creció rápidamente y grandes compañías han entrado en el espacio de las redes sociales en Internet. Por ejemplo, Google lanzó Orkut el 22 de enero de 2004. Otros buscadores como KaZaZz! y Yahoo! crearon redes sociales en 2005. En estas comunidades, un número inicial de participantes envían mensajes a miembros de su propia red social invitándoles a unirse al sitio. Los nuevos participantes repiten el proceso, creciendo el número total de miembros y los enlaces de la red. Los sitios ofrecen características como actualización automática de la libreta de direcciones, perfiles visibles, la capacidad de crear nuevos enlaces mediante servicios de 
presentación y otras maneras de conexión social en línea. Las redes sociales también pueden crearse en torno a las relaciones comerciales.

Aplicaciones Web. En la ingeniería de software se denomina aplicación Web a aquellas aplicaciones que los usuarios pueden utilizar accediendo a un servidor Web a través de Internet o de una intranet mediante un navegador. En otras palabras, es una aplicación software que se codifica en un lenguaje soportado por los navegadores Web, en la que se confía la ejecución al navegador. Las aplicaciones Web son populares debido a la practicidad del navegador Web como cliente ligero, a la independencia del sistema operativo, así como a la facilidad para actualizar y mantener aplicaciones Web sin distribuir e instalar software a miles de usuarios potenciales. Existen aplicaciones como los webmails, wikis, weblogs, tiendas en línea y la propia Wikipedia que son ejemplos bien conocidos de aplicaciones Web.

Sobre la Web 3.0, cabe reseñar que se fundamenta en el colectivo de la Web Semántica, búsquedas en lenguaje natural, data-mining, el aprendizaje automático y la asistencia de agentes; todo ellos conocido como técnicas de la inteligencia artificial. Podemos estar viviendo la Web 3.0 si la consideramos como la extensión Web que se basa en la Web Geoespacial o la Web 3D.

\section{DISPERSIÓN Y VOLATILIDAD}

Si hay tres términos que pueden definir claramente la información en la Web 2.0 éstos son: dispersa, volátil y efímera.

Podemos decir que la Web 2.0 se ha definido como una conversación, una conversación dispersa entre muchos lugares, en redes sociales, en blogs, en vblogs, en portales propios de prensa, etc. La gran mayoría de las veces la repercusión que esta información puede provocar se ha extendiendo y dispersado por las tecnologías de la Web 2.0, perdiendo así su eco. El poder de las redes sociales genera comentarios cuyo feedback, a veces, no se realiza hacia el lugar que generó el contenido o la información.

Podemos decir pues, que la noticia de prensa o el post del blog, ya no aúnan al pie de su página todas las conversaciones que se han generado de esta noticia. Probablemente la difusión de esa noticia se haya dado en gran parte por alguna red social como Twitter y esos comentarios que se generaban antes en el pie de la entrada del Blog se están generando como respuestas en esta red social, o retuiteos; sin olvidar que además de haber podido ser difundida por la red social Twitter puede haberse enlazado en la red social de Facebook, creando así un hilo de conversación más, y otro hilo más al estar también publicada en Google+, pueden darse todas las posibilidades a la vez o ninguna; la información puede estar publicada en las tres redes sociales, pero debemos recordar que estos medios, a día de hoy, son meros contenedores de hallazgos ajenos, que lo máximo que pueden hacer es dispersar la información e incluso, hacerla parasitar. El objetivo que las redes sociales tienen claro sobre la información es el factor de inmediatez de la misma. Pero, ¿qué ocurre con la recuperación de esa noticia de prensa pasados unos meses?, ¿qué ocurre con la recuperación de esa dispersión?, ¿cómo se puede solucionar? 
Es un debate largo e intenso, ya que a la gran mayoría de bloggers nos preocupa un cierre sistemático de alguna red social. Hace años Google lanzó SideWiki, con él podíamos permitirnos anotar cualquier comentario a modo universal y a la vez mantenerlos localizados. Pero, posteriormente, Google decidió cerrarlo al lanzar Google+; de la misma manera si esta red social crece y crece como se presiente, el resto de redes sociales también pueden desaparecer y por ende lo efímero y delicado de la información y feedbacks que generemos en ellas.

Podemos llegar a pensar que la respuesta a esta volatilidad, dispersión y a el carácter efímero de la información en la Web 2.0 puede estar en los propios blogs, pero para ello también han de mantenerse activos, o el blog o las entradas.

\section{ARAÑÁPOLIS O GOOGLE CONTRA EL MUNDO}

Corría el mes 11 del año 2011, Google mejora su algoritmo, esta mejora no supone un cambio, sino 30; de estos 30 cambios, el más importante es primar los enlaces más recientes cuando de una búsqueda sobre actualidad se trate. La intención es dar frescura a los enlaces, suponiendo un ligero cambio de alrededor del $38 \%$ en los resultados de las búsquedas.

"Si el internauta busca sobre los Oscar, Google entenderá que espera ver los datos más nuevos y no una rememoración de lo que ocurrió hace 50 años." (El País Digital, 04/11/2011).

El objetivo de Google siempre ha sido la primacía como buscador, pero siempre hemos oído hablar de las amenazas de Google, incluyendo su particular valoración y selección de los resultados de búsqueda, y de si ésta satisface adecuadamente las necesidades de información de los usuarios. Sabemos que la recuperación de información no es neutral, por lo tanto debemos aclarar que los problemas al recuperar información a veces no sólo los provoca Google. A veces no es un problema de recuperación, sino de consulta. Cuando estos problemas de consulta aparecen siempre reclamamos a Google que se acerque un poco a la búsqueda social, al lenguaje natural, a la web semántica. Pues, a veces al aclamar al cielo, puede llover. Y llovió.

Google decide acercarse a lo social; el gigante de las búsquedas se lanza a por la Web contextual social, a por la búsqueda social, después de probar la incorporación de lo social, probando características, mecanismos de medida de la confianza a sus resultados. Primero fueron las redes sociables como revolución social, ahora son las búsquedas las que tiñen de sociabilidad la información.

La aparición de Google Search Plus Your World revoluciona todo el mundo de las búsquedas, no sólo pretende dar mayor cobertura a la Web social sino que pretende dotar de mayor control sobre los resultados para los usuarios. A partir de ahora, las arañas de Google no sólo van a rastrear entre miles de millones de webs, sino que también lo harán entre los textos, fotos y comentarios que la gente colgará en la red social de Google, Google+.

Parece una buena idea humanizar el buscador indexando también los contenidos de la red social, así Google se acerca, como decíamos a la Web 2.0 o a la Web contextual social, pero ¿dónde están las consecuencias? 
Si volvemos a los cambios que Google implementó en noviembre de 2011, cabe recordar las palabras de Eric Schmidt que dejaban bien claro que "Google no hace nada para bloquear el acceso a ninguno de sus competidores y a otras fuentes de información".

Sin embargo, la recuperación se ve afectada, puesto que Google quiere ofrecer un mundo parcial, al indexar el contenido de una sola red social, la suya (primer símbolo de partidismo y monopolio). Además, pese a que Google sigue intentando demostrar que aparecen resultados de terceros en las búsquedas, somos conscientes, o debemos serlo, de la no indexación de caracteres especiales por parte de Google; así pues, se deja fuera de los resultados a Twitter por incluir caracteres tales como \# o @, sin embargo reconocen indexar alrededor de 3000 millones de páginas de esta red social. Google deberá pues definir claramente qué es para él la Información Social Relevante.

Hablamos de la desaparición de neutralidad, de nuevo la primacía de Google, y cómo éste favorece a sus aplicaciones y al crecimiento de Google+, un claro abuso de posición dominante. Hablamos de una red social que ya ha llegado a los 90 millones de usuarios, pero que el tiempo medio de navegación en esa red es de 5 minutos al mes, está claro que indexar ese contenido sería importante si se tratara de un foco de información, un hervidero, ¿pero conocemos quiénes y cuántos usan Google+? 


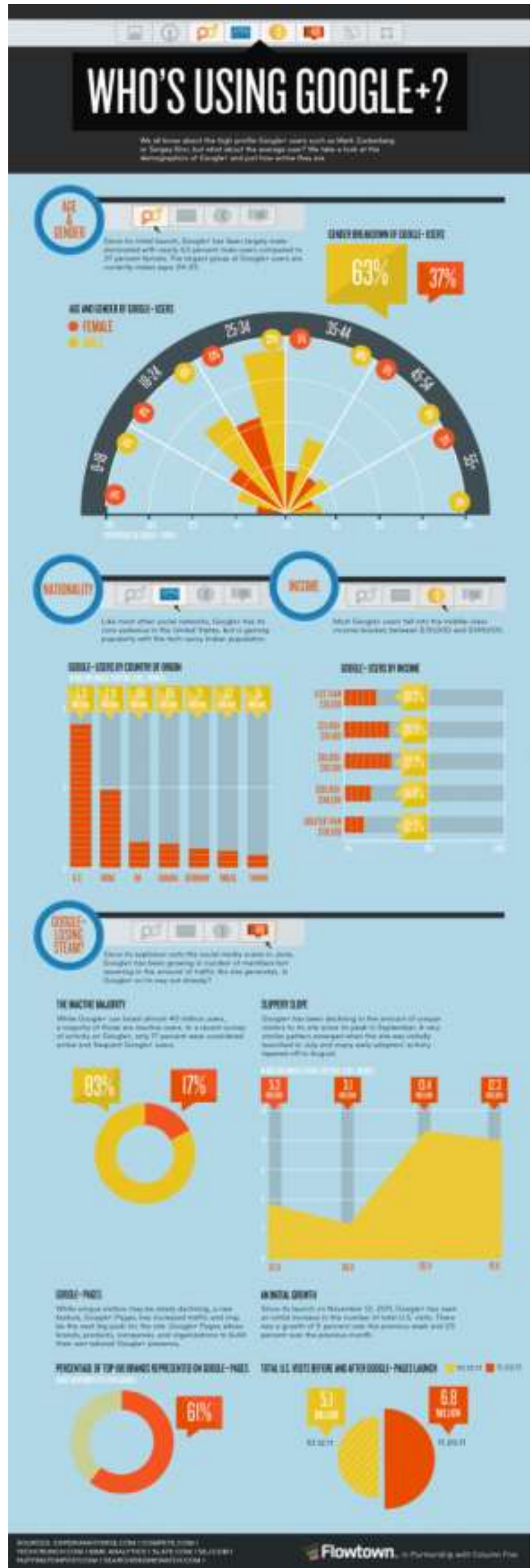

Fuente: Flowtown.com 
Search Plus Your World según Google incluye 3 nuevas funcionalidades al buscador Google:

Resultados Personales, que permitirá encontrar información justo para los usuarios, como fotos y publicaciones en Google+.

Perfiles dentro de las Búsquedas, donde se va a permitir encontrar de manera inmediata a personas que conocemos o bien, que pudiéramos estar interesados en conocer.

Personas y Páginas, que ayudará a encontrar los perfiles y páginas en Google+ relacionados con una área de interés específica; y permitirá su seguimiento a golpe de clic. Además, detrás de cada búsqueda, normalmente hay una comunidad más grande.

Pero, volvemos al inicio, en lugar de extraer contenidos de varias redes sociales, Google Search Plus depende mucho de su propia red, Google+.

Podemos decir en este momento que estamos frente a una búsqueda que ni es real ni es tan social como Google pretende hacernos ver. Obviamente está condicionada a una plataforma sin volumen, sin aceptación voluntaria por el usuario, una búsqueda social que puede representar el 3,5\% sobre la Audiencia Mundial según comScore; al trabajar la búsqueda sobre un mundo parcial, deberíamos cambiar el nombre de búsqueda social a búsqueda asocial, de una comunidad selecta y reducida.

Las políticas comerciales de Google inciden negativamente en la pertinencia y relevancia de los resultados, perjudicando a la búsqueda objetiva. La relevancia ya no consiste en ofrecer los resultados que corresponden con lo que buscamos, sino lo que las herramientas que empleamos para buscar información estiman que es lo que más se ajusta a nuestras supuestas preferencias, junto a aquello que creen que nos puede interesar en virtud de nuestro perfil: noticias, fotos, vídeos, etc. No se ofrece lo que buscamos, sino lo que se cree que buscamos [Merlo Vega, 2012]. 


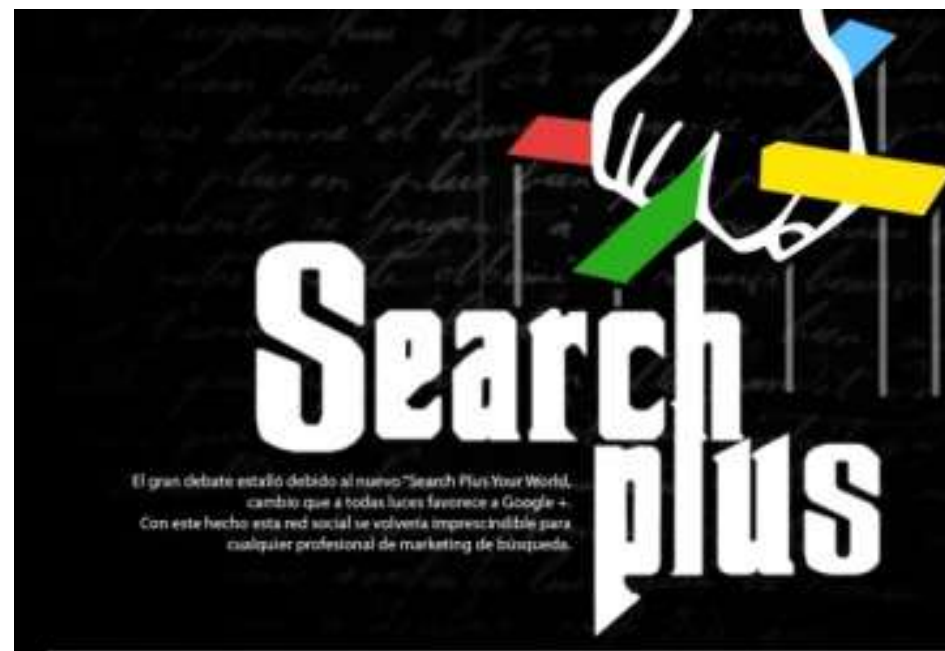

EN LOS RESULTADOS DE BÚSQUEDA SÓLO OFRECEN SUGERENCIAS DE GOOGLE +

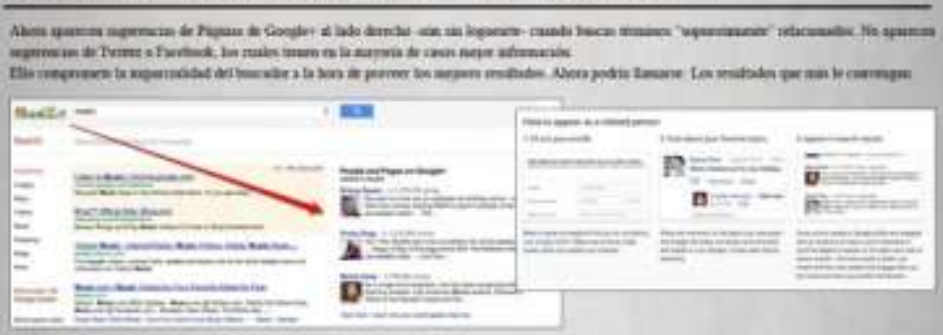

GOOGLE + SE KACE CARCO DE LAA CAJA DE BOSQUEDAS

GOOGLE + SE VUELVE UN

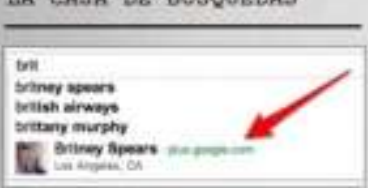

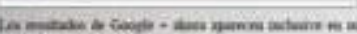

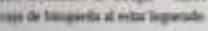
DEBER PARA LOS MARKETEROS

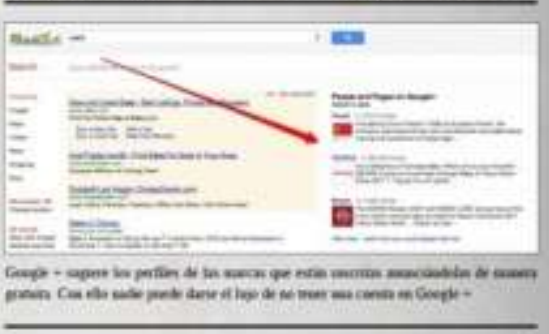

¿GOOGLE

ESTÁ BROMEANDO?

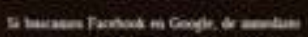

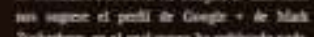

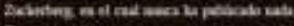

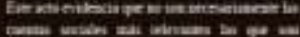

andest

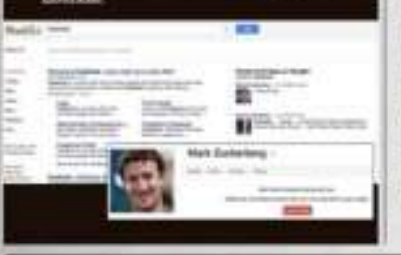

GUERRA ENTRE GOOGLE Y TWITTER

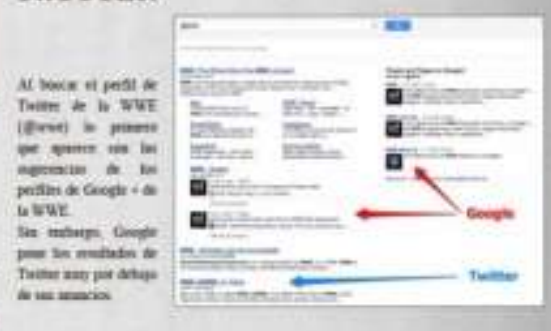

ancinas

$M$

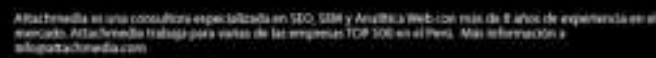

Dethihmatas

Fuente: http://www.attachmedia.com 


\section{CONCLUSIONES: HACIA UNA RECUPERACIÓN OBJETIVA.}

Frente a los ataques directos de Google, sólo nos queda luchar, después de haber aprendido que si no estamos en Google no somos nada, debemos desaprenderlo para poder obtener unos resultados objetivos y relevantes para nuestras consultas, que desde los comienzo ya se han visto dirigidas con, por ejemplo, la geolocalización en el buscador.

Para llegar a nuestra meta de recuperación objetiva, debemos rescatar el concepto de anonimato, así pues, tener en cuenta no abrir sesión alguna de Google al realizar una consulta; con una identificación previa, sabemos que las consultas se van a ir almacenando y paulatinamente los resultados se van a ajustar al historial de búsquedas.

Volver a hacer uso de la búsqueda avanzada, combinar los términos de búsqueda para ajustar los resultados como recurso esencial, que se trate de un sistema clásico no significa que sea obsoleto. Desenfundar los operadores, los de precisión para una recuperación pertinente. Hacer uso de los filtros por área geográfica, lengua, fecha,... Usar la búsqueda textual. Conseguir una despersonalización de los resultados borrando la información personal. Eliminar el Historial del navegador. Vaciar la memoria caché e incluso utilizar fuentes específicas para cada tipo de información, no sólo buscadores.

Cierto es que disponemos de las herramientas para esta Web 2.0, en materia de búsqueda y recuperación de información, y que la proliferación es real e inminente. $Y$ aunque parece paradójico, es este momento cuando la figura del documentalista no queda relegada a un segundo plano, sino que ha de verse como protagonista y figura principal en este proceso de búsqueda y recuperación de información, ya que deberá discernir cómo realizar búsquedas de información y hallar el verdadero equilibrio entre silencio y ruido documental. 


\section{REFERENCIAS BIBLIOGRÁFICAS}

POLO, Juan Diego. Google presenta Search Plus Your World, una nueva forma de usar su buscador [en línea]. Disponible en: http://wwwhatsnew.com/2012/01/10/google-presentasearch-plus-your-world-una-nueva-forma-de-usar-su-buscador/. [Consulta: 14 de febrero de 2012].

GOOGLE. El Blog Corporativo de Google para América Latina: Blog en español sobre nuestros asuntos públicos: sociedad, cultura y política [en línea]. Disponible en: http://googleamericalatinablog.blogspot.com.es/2012/01/presentando-search-plus-yourworld.html. [Consulta: 14 de febrero de 2012].

FUSCO, P.J. How Google Search Plus Your World is Changing SEO [en línea]. Disponible en: http://searchenginewatch.com/article/2141420/How-Google-Search-Plus-Your-World-isChanging-SEO. [Consulta: 14 de marzo de 2012].

INTERNET ADVANTAGE. Google Search, Plus Your World y Adwords [en línea]. Disponible en: http://www.internetadvantage.es/sem/google-search-plus-your-world/. [Consulta: 14 de marzo de 2012].

MERLO-VEGA, José Antonio. Rebelarse contra la relevancia: cómo encontrar lo que buscamos y no lo que Google cree que buscamos [en línea]. Disponible en: http://www.thinkepi.net/rebelarse-contra-la-relevancia-como-encontrar-lo-que-buscamos-yno-lo-que-google-cree-que-buscamos/. [Consulta: 24 de marzo de 2012].

REIG, DOLORS. Content Curator, Intermediario del conocimiento: nueva profesión para la web 3.0 [en línea]. Disponible en: http://www.dreig.eu/caparazon/2010/01/09/content-curatorweb-3/. [Consulta: 24 de marzo de 2012].

EuropaPress. Google se prepara para "pensar" como un humano con la búsqueda semántica. [en línea]. Disponible en: http://www.europapress.es/portaltic/internet/noticia-googleprepara-pensar-humano-busqueda-semantica-20120315123338.html . [Consulta: 20 de marzo de 2012].

SEGOVIA, Adrián. Google inventa la búsqueda 'asocial' [en línea]. Disponible en: http://blogs.elpais.com/estrategia-digital/2012/01/google-inventa-la-busqueda-asocial.html/ [Consulta: 15 de marzo de 2012].

SINGHAL, Amit. (Google Fellow). Search, plus Your World [en línea]. Disponible en:http://googleblog.blogspot.com/2012/01/search-plus-your-world.html. [Consulta: 2 de marzo de 2012].

REIG, Dolors. Google Search plus Your World: La web contextual social [en línea]. Disponible en: http://www.dreig.eu/caparazon/2012/01/11/pregunta-a-tu-mundo. [Consulta: 2 de marzo de 2012]. 
TRAMULLAS, Jesús. Caffeine, el nuevo índice para las búsquedas de Google [en línea]. Disponible en: http://tramullas.com/2010/06/10/caffeine-nuevo-indice-para-las-busquedasde-googe. [Consulta: 2 de marzo de 2012].

COMPUTERWORLD. Facebook y Twitter lanzan herramienta de búsqueda para Google [en línea]. Disponible en: http://www.cwv.com.ve/?p=7034. [Consulta: 2 de marzo de 2012].

PARFENI, Lucian. Google finalmente responde a las críticas contra la función Search Plus Your World [en línea]. Disponible en: http://news.softpedia.es/Google-finalmente-responde-a-lascriticas-contra-la-funcion-Search-Plus-Your-World-249418.html. [Consulta: 10 de marzo de 2012]. 